\title{
Caracterização técnica e desempenho hidráulico de quatro gotejadores autocompensantes utilizados no Brasil
}

\author{
Technique characterization and hydraulic performance \\ of four self-compensating drippers used in Brazil
}

\author{
Alexandre Barcellos Dalri ${ }^{I^{*}}$ Carlos Jesus Baca Garcia ${ }^{I I}$ José Renato Zanini \\ Rogério Teixeira de Faria ${ }^{\mathrm{I}}$ Luiz Fabiano Palaretti ${ }^{\mathrm{I}}$
}

RESUMO

Este trabalho teve por objetivo caracterizar $e$ avaliar o desempenho de quatro modelos de gotejadores autocompensantes, frequentemente utilizados nos sistemas de irrigação por gotejamento no Brasil. Para realização do estudo, foi tomada uma amostra de 25 gotejadores de cada modelo. $\mathrm{Na}$ avaliação dos respectivos tubos emissores, foram determinados os seguintes parâmetros: (a) vazão média, (b) coeficiente de variação de fabricação, (c) espessura da parede (d), equação característica do gotejador, (e) diâmetro interno do tubo, (f) espaçamento entre emissores, (g) resistência à pressão com água na temperatura ambiente e à temperatura de $40^{\circ} \mathrm{C}$, e (h) resistência do tubo à tração com tensão de $160 \mathrm{~N}$ e $180 \mathrm{~N}$. As análises mostraram coeficientes de variação de fabricação inferiores a 0,045 para todos os gotejadores (0,07 é o limite máximo, segundo a ABNT NBR ISO 9261). Os expoentes $x$ da equação pressão versus vazão variaram de 0,0082 a 0,0691, classificando-os como autocompensantes. Pelos resultados obtidos, é possível afirmar que nos produtos ensaiados, os quais são utilizados no Brasil pelos irrigantes, apresentaram desempenho hidráulico satisfatório, de acordo com a norma.

Palavras-chave: avaliação, emissor, irrigação por gotejamento.

\section{ABSTRACT}

This study aimed to characterize and evaluate the performance of four models self-compensating drippers, commonly used in drip irrigation systems in Brazil. To perform the study, it was taking a sample of 25 emitters of each model. Evaluating their tubes emitting, the following parameters were determined: (a) mean discharge, (b) coefficient of manufacturing variation (c) wall thickness, (d) characteristic equation of the dripper, (e) internal diameter of the pipe, (f) spacing between emitters, $(g)$ resistance water pressure at ambient temperature and to $40^{\circ} \mathrm{C}$, and (h) the tensile strength of the pipe with tension of $160 \mathrm{~N}$ and 180 $N$. The analysis showed variation coefficient of less than 0.045 for all manufacturing drippers (0.07 is maximum according to ABNT
NBR ISO 9261). The exponents $x$ of the equation pressure versus discharge ranged from 0.0082 to 0.0691 , classifying them as selfcompensating. From the results obtained it is clear that the tested products, which are used in Brazil and used by irrigators had a satisfactory hydraulic performance in accordance with the standard.

Key words: evaluation, emitter, drip irrigation.

\section{INTRODUÇÃO}

Dos grandes usuários dos recursos hídricos, a irrigação é o segmento que mais consome água. Em algumas regiões, o consumo de água por meio da irrigação pode superar $70 \%$ do total utilizado. Para maximizar o uso desse recurso, deve-se melhorar a eficiência da aplicação e, para isso, os dispositivos reguladores, controladores de aplicadores de água devem possuir qualidade compatível com o objetivo a que se propõem (MANTOVANI et al., 2012).

Os emissores gotejadores constituem um dos principais componentes de irrigação por gotejamento. Os materiais utilizados no processo de fabricação, bem como o próprio processo de fabricação, fazem com que os emissores de um mesmo modelo não sejam exatamente iguais entre si e, como consequência, podem ocasionar diferentes vazões, mesmo quando trabalhando na mesma pressão de serviço (FRIZZONE \& DOURADO NETO, 2003). Em relação às características dos tubos gotejadores, a espessura da parede dos tubos gotejadores varia muito, dependendo da durabilidade esperada e se o sistema será enterrado

\footnotetext{
'Departamento de Engenharia Rural, Faculdade de Ciências Agrárias e Veterinárias (FCAV), Universidade Estadual Paulista (UNESP), 14884-900, Jaboticabal, SP, Brasil. E-mail: dalri@fcav.unesp.br. *Autor para correspondência.

"Facultad de Agronomía y Zootecnia, Universidad Nacional de San Antonio Abad del Cusco (UNSAAC), Cusco, Perú. Recebido 04.06.14 Aprovado 14.11.14 Devolvido pelo autor 15.03.15 CR-2014-0860.R1
} 
(ANDRADE \& BORGES JUNIOR, 2008). Quanto mais delgadas forem as suas paredes, menor o custo de produção. Os espaçamentos também são bem variados, de $0,1 \mathrm{~m}$ até $1,0 \mathrm{~m}$ entre emissores. Os gotejadores podem ser de fluxo turbulento e não apresentam dispositivos de compensação de pressão, ou emissores autocompensantes, que dispõem de dispositivos de auto regulagem da vazão (PIZARRO CABELLO, 1996; RODRIGO LOPEZ et al., 1997; GOMES, 1997).

Toda dificuldade no projeto construtivo de um tubo gotejador reside no seguinte problema: os gotejadores devem ser capazes de dissipar a pressão disponível e aplicar vazões pequenas, constantes e uniformes, em pontos discretos sistematicamente localizados (PIZARRO CABELLO, 1996; RODRIGO LOPEZ, 1997). Por outro lado, a pressão de serviço dos emissores não deve ser alta, para reduzir o consumo de energia e também não deve ser muita baixa, para não comprometer seu desempenho hidráulico. Essas condições conduzem à fabricação de emissores gotejadores com reduzido diâmetro da seção de escoamento. Por outro lado, a seção de escoamento do emissor deve ser a maior possível, com o objetivo de evitar obstruções.

Para melhorar a uniformidade e a eficiência da aplicação de água pela irrigação, bem como permitir aos projetistas a construção de linhas laterais mais longas, foram desenvolvidos os gotejadores autocompensantes. Esses emissores possuem, de uma maneira geral, um custo mais elevado, porém realizam autorregulagem da vazão quando ocorre variação da pressão hidráulica no interior da linha.

Há, no mercado brasileiro, diversos modelos de gotejadores autocompensantes, porém os irrigantes, bem como os projetistas, desconhecem suas principais características de desempenho. O objetivo deste trabalho foi caracterizar tecnicamente e avaliar o desempenho hidráulico de quatro modelos de tubos emissores autocompensantes usados no Brasil, uma vez que várias especificações técnicas não se encontram disponíveis nos catálogos, nem nas embalagens dos produtos fabricados.

\section{MATERIAL E MÉTODOS}

$\mathrm{O}$ experimento foi conduzido no laboratório de hidráulica da UNESP/FCA, Botucatu, SP. Foram utilizados quatro modelos de gotejadores autocompensantes, sendo eles: Amanco Drip PC, Naan PC, Ram e Twin Plus.
Para avaliar o desempenho hidráulico e técnico dos tubos gotejadores autocompensantes, foram analisados os seguintes parâmetros: (a) vazão média $\left(\mathrm{q}_{\mathrm{m}}\right)$, (b) coeficiente de variação de fabricação (CVF), (c) espessura média da parede nas duas extremidades $\left(\mathrm{e}_{1}, \mathrm{e}_{2}\right)$, (d) equação característica do gotejador, (e) diâmetro interno do tubo $\left(\mathrm{D}_{\mathrm{i}}\right),(\mathrm{f})$ espaçamento entre emissores $\left(\mathrm{S}_{\mathrm{e}}\right),(\mathrm{g})$ resistência à pressão com água na temperatura ambiente $\left(\mathrm{R}_{\mathrm{t} 1,2}, \mathrm{e}\right.$ $\left.\mathrm{R}_{\mathrm{t} 1,8}\right)$ è temperatura de $40^{\circ} \mathrm{C}\left(\mathrm{R}_{\mathrm{t} 40}\right)$, resistência do tubo à tração com tensão de $160 \mathrm{~N}\left(\mathrm{R}_{\mathrm{t} 160}\right)$ e $180 \mathrm{~N}\left(\mathrm{R}_{\mathrm{t} 180}\right)$.

$\mathrm{A} \mathrm{q}_{\mathrm{m}}$ observada em 25 emissores de cada modelo, foi avaliada por pesagem e transformada em volume, sendo que o tempo de coleta para cada ensaio foi de 5 minutos. Antes da coleta do volume, os tubos gotejadores passaram por processos de pressurização, denominado de condicionamento da amostra. Os procedimentos para condicionamento seguiram os seguintes passos: foi ajustada a pressão mínima de trabalho, mantida por 3 minutos e, posteriormente, ajustada à pressão máxima de trabalho por mais 3 minutos, sucessivamente até os 18 minutos. Logo após, ajustou-se a pressão nominal até que o tempo total de condicionamento completasse uma hora. Cessado o condicionamento e mantendo-se a pressão, coletou-se o volume. Foram realizadas quatro repetições na avaliação da $\mathrm{q}_{\mathrm{m}}$. A pressão média ou a nominal adotada foi a recomendada pelo fabricante de cada modelo.

O coeficiente de variação de fabricação (CVF) é decorrente do projeto do gotejador, material utilizado em sua fabricação e da qualidade com que o gotejador é fabricado. Segundo KELLER \& BLIESNER (1990), seu valor é calculado pela razão do desvio padrão (s) da amostra pela $\mathrm{q}_{\mathrm{m}}$, geralmente expresso em porcentagem, $\mathrm{CVF}=\left(\frac{\mathrm{s}}{\mathrm{q}_{\mathrm{m}}}\right) 100$.

$\mathrm{s}$ - desvio padrão da amostra, $\mathrm{L} \mathrm{h}^{-1}$; e

$\mathrm{q}_{\mathrm{m}}$ - vazão média da amostra, $\mathrm{L} \mathrm{h}^{-1}$.

A equação característica do emissor é expressa pela equação $\mathrm{q}=\mathrm{k} \mathrm{H}^{\mathrm{x}}$ (FRIZZONE et al., 2012), sendo:

$\mathrm{q}$ - vazão do gotejador, $\mathrm{L} \mathrm{h}^{-1}$;

$\mathrm{H}$ - pressão de trabalho; $\mathrm{kPa}$;

$\mathrm{k}$ - coeficiente da equação do emissor, adimensional; e $\mathrm{x}$ - expoente de fluxo, adimensional.

Com o auxílio de um micrômetro com precisão de $0,01 \mathrm{~mm}$, determinou-se a espessura da parede $\left(\mathrm{e}_{1}, \mathrm{e}_{2}\right)$ do tubo gotejador em quatro 
pontos equidistantes em duas seções transversais ao comprimento do tubo, ou seja, foram tomadas quatro medidas em cada extremidade do tubo, sendo $\mathrm{e}_{1}$, bem como $\mathrm{e}_{2}$, a média das quatro leituras. Para determinação do diâmetro interno (Di) do tubo emissor, inseriu-se em uma das extremidades do tubo gotejador uma ferramenta cônica com ângulo de $10^{\circ}$. Marcou-se na ferramenta o círculo feito pela extremidade do tubo, medindo-se, em seguida, o diâmetro do tubo. O espaçamento entre emissores (Se) foi determinado com auxílio de uma trena.

$\mathrm{Na}$ avaliação da resistência do tubo à pressão em temperatura ambiente, os tubos gotejadores ficaram gotejando por 60 minutos à pressão de 1,2 vez a pressão máxima, denominado de $\left(\mathrm{R}_{\mathrm{t} 1,2}\right)$, determinando-se a vazão nos gotejadores após esse condicionamento. $\mathrm{Na}$ avaliação à pressão de 1,8 vez à pressão máxima, $\left(\mathrm{R}_{\mathrm{t} 1,8}\right)$, outros novos gotejadores passaram pelo mesmo condicionamento, porém com pressão $80 \%$ superior à pressão máxima. Após este condicionamento, fez-se a coleta da vazão dos emissores sujeitos à pressão nominal. A pressão máxima adotada para cada modelo de gotejador autocompensante foi a indicada pelo fabricante. $\mathrm{Na}$ avaliação da resistência à pressão com água na temperatura de $40^{\circ} \mathrm{C}\left(\mathrm{R}_{\mathrm{t} 40}\right)$, delimitou-se um trecho com 3 emissores de cada modelo de tubo. $\mathrm{O}$ tubo gotejador foi colocado num recipiente com água aquecida $\left(38^{\circ} \mathrm{C}\right.$ a $\left.43^{\circ} \mathrm{C}\right)$, permanecendo em funcionamento por 60 minutos. Depois desse tempo foi realizada a determinação da vazão dos gotejadores em temperatura ambiente e sujeito à pressão nominal. O condicionamento da amostra com água aquecida, bem como o teste, foi realizado na pressão nominal de cada modelo de tubo gotejador.
No ensaio de resistência à tração, o qual tem como objetivo conhecer se o tubo pode ser considerado como reutilizável, utilizou-se de dois trechos do tubo gotejador de cada fabricante. Aplicaram-se duas tensões, $160 \mathrm{~N}$ e $180 \mathrm{~N}$, em um seguimento de $150 \mathrm{~mm}$ do tubo gotejador, tomando-se o cuidado de incrementar a tração lentamente, de maneira a não danificar as amostras. A tração foi mantida por 15 minutos. Após esse tempo, retiraram-se os tubos do dispositivo de tração e avaliou-se o alongamento do tubo, bem como a vazão na pressão nominal. A água utilizada no ensaio foi potável, proveniente do abastecimento da instituição. A água apresentou $2,1 \mathrm{mg} \mathrm{L}^{-1}$ de resíduos sólidos, estando dentro do limite aceitável de até $25 \mathrm{mg} \mathrm{L}^{-1}$ (ABNT, 2006). Os dados foram tabulados e as equações de regressão foram obtidas com o auxílio de planilha eletrônica.

\section{RESULTADOS E DISCUSSÃO}

a) Vazão média - Na tabela 1, encontram-se os valores obtidos da $\mathrm{q}_{\mathrm{m}}$ para os quatro modelos de tubo gotejador analisados. Podem ser observados os valores medidos e as diferenças percentuais entre as vazões fornecidas pelos fabricantes (Nominal) em relação aos valores obtidos no ensaio (vazão média observada). A maior variação observada na vazão média dos gotejadores analisados foi para o modelo Twin Plus, atingindo o valor positivo de $6,56 \%$. Porém, esse valor está dentro do limite aceito pela norma brasileira NBR ISO 9261 (ABNT, 2006). Pode-se afirmar que os produtos avaliados estão dentro das especificações exigidas pela norma técnica. Dos quatro modelos avaliados, apenas um apresentou vazão inferior ao informado pelo fabricante (modelo Naan PC), porém dentro do permitido pela norma técnica brasileira.

b) Coeficiente de variação de fabricação (CVF) - Os valores obtidos do efeito dos fatores

Tabela 1 - Variáveis analisadas dos tubos gotejadores autocompensantes. Vazão média ( $\mathrm{q}_{\mathrm{m}}$ ) observada em função da pressão nominal de serviço, coeficiente de variação de fabricação (CVF) e espessura da parede do tubo gotejador (e), sendo $\mathrm{e}_{1}$ e $\mathrm{e}_{2}$ as extremidades do tubo gotejador.

\begin{tabular}{|c|c|c|c|c|c|c|c|c|}
\hline \multirow{2}{*}{ Modelo } & \multirow{2}{*}{ Pressão nominal (kPa) } & \multicolumn{2}{|c|}{------ $q_{m}\left(L_{h}^{-1}\right)$------ } & \multirow{2}{*}{ Variação $(\%)^{1}$} & \multirow{2}{*}{$\mathrm{CVF}^{2}$} & \multicolumn{3}{|c|}{ - } \\
\hline & & Nominal & Observada & & & Informada & $e_{1}$ & $e_{2}$ \\
\hline Amanco & 250 & 2,30 & 2,33 & $+1,30$ & 0,045 & 0,90 & 1,04 & 1,75 \\
\hline Naan PC & 225 & 3,80 & 3,73 & $-1,84$ & 0,029 & 0,90 & 0,94 & 0,93 \\
\hline Ram & 175 & 2,30 & 2,36 & $+2,61$ & 0,019 & 1,00 & 1,08 & 1,07 \\
\hline Twin Plus & 250 & 1,83 & 1,95 & $+6,56$ & 0,035 & 1,00 & 1,17 & 1,18 \\
\hline
\end{tabular}

${ }^{1}$ Variação permitida $\pm 7 \%$ do valor informado em relação ao medido; ${ }^{2}$ CVF não deve exceder 0,07 ; "e" medida deve ser maior que $90 \%$ da informada (NBR ISO 9261:2006). 
construtivos para todas as marcas avaliadas, expresso pelo coeficiente de variação de fabricação (CVF), estiveram inferiores a 0,07 (limite máximo segundo a norma). Portanto, pode-se inferir que todos os produtos encontrados no mercado brasileiro e avaliados neste trabalho podem ser considerados de ótima qualidade do ponto de vista do processo de fabricação (Tabela 1). PRADO et al. (2014), analisando as características hidráulicas de gotejadores, também encontraram valores do CVF abaixo de $7 \%$ para os modelos estudados. Um ótimo controle de qualidade no processo de fabricação dos emissores é uma das metas dos fabricantes, pois os emissores que apresentam valores elevados do CVF proporcionam alta variação da vazão entre eles (SOUZA et al., 2012), o que não é indicado para a irrigação localizada, principalmente quando se realiza a fertirrigação.

c) Espessura da parede do tubo - Em relação à espessura da parede dos tubos gotejadores, pode-se afirmar que todas as marcas comerciais estão dentro das especificações da norma NBR ISO 9261 para este item. Os dados médios obtidos nas leituras estão apresentados na tabela 1. Observa-se que os quatro modelos analisados apresentam espessura da parede superior à informada, beneficiando diretamente os irrigantes, usuário dos produtos.

d) Equação característica do gotejador - A figura 1 apresenta o comportamento hidráulico dos modelos de gotejadores autocompensantes estudados com suas respectivas equações características e coeficientes de determinação $\left(\mathrm{R}^{2}\right)$. Pela norma técnica referenciada (NBR ISO 9261), quando o valor do expoente da equação característica do emissor x é maior do que 0,2 , considera-se que este emissor não é autocompensante. Pode-se observar, na figura 1, que, nos quatro modelos analisados, todos apresentam valores inferiores a 0,2 , podendose afirmar que esses produtos são autocompensantes, conforme manifestam seus fabricantes. $\mathrm{O}$ expoente $\mathrm{x}$ da equação característica do gotejador, que expressa a sensibilidade à variação de pressão variou de 0,0691 para o modelo Twin Plus a 0,0082 para o modelo Ram. Dos modelos estudados, o Ram foi o que apresentou menor sensibilidade à flutuação da pressão hidráulica. Para uma variação da pressão de $100 \%$ no gotejador autocompensante Ram, ocorre um pequeno aumento na variação da vazão, da ordem de $0,55 \%$, comprovando o efeito da compensação da variação de pressão.

e) Diâmetro interno do tubo - Na tabela 2, estão apresentados os valores do Di informados
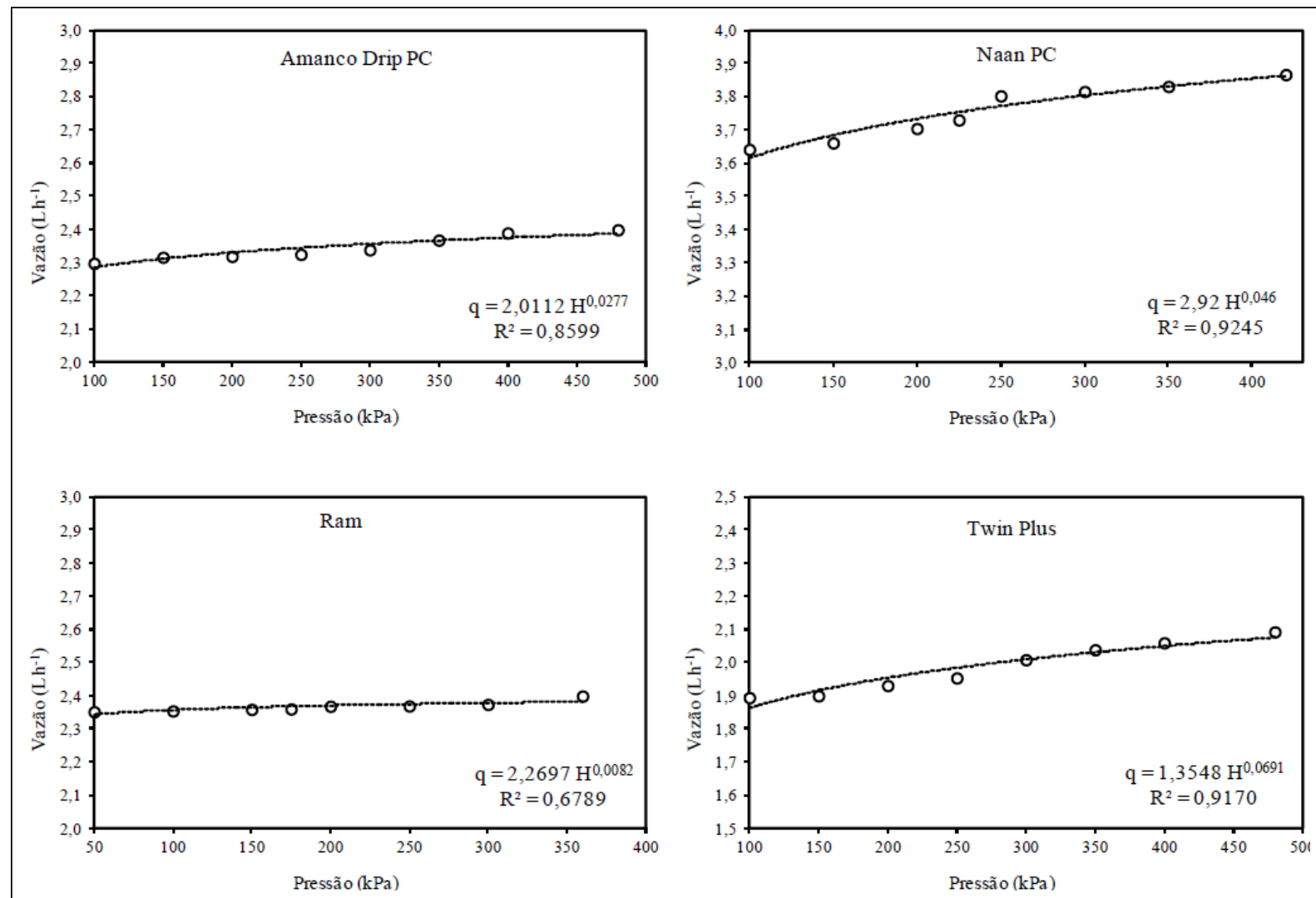

Figura 1 - Equações potenciais da relação vazão $(q)$ versus pressão $(H)$ para os quatro modelos de gotejadores autocompensantes avaliados.

Ciência Rural, v.45, n.8, ago, 2015. 
Tabela 2 - Diâmetros internos (Di) e espaçamento entre emissores (Se), informados e medidos no ensaio experimental.

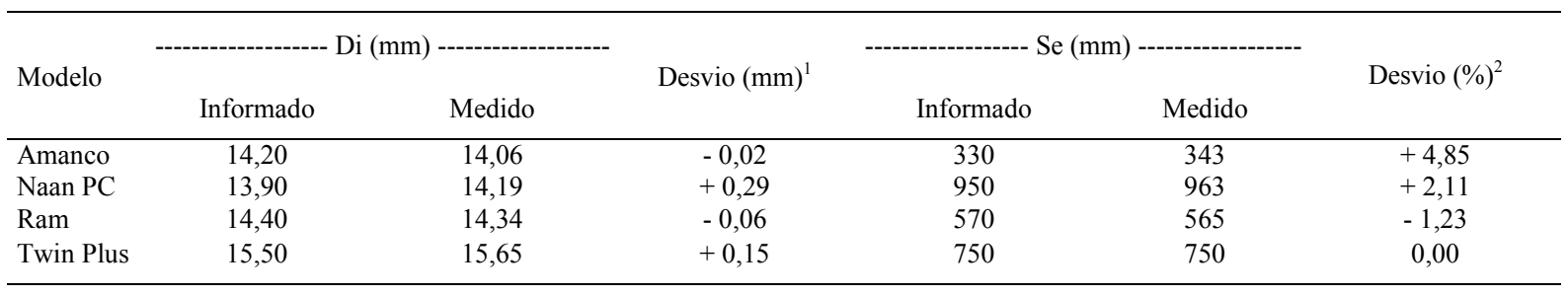

${ }^{1}$ Desvio do Di (aceitável $\left.\pm 0,3\right) ;{ }^{2}$ desvio do Se (aceitável $\pm 5 \%$ ), (NBR ISO 9261:2006).

pelo fabricante e medidos neste estudo. Pode-se observar que todos os tubos emissores estão dentro da faixa aceitável da norma. Essa variável analisada apresenta novamente a excelente qualidade dos tubos gotejadores utilizados na irrigação brasileira. A padronização do Di nos modelos dos tubos gotejadores proporciona facilidade de montagem aos irrigantes e possível redução dos vazamentos ocorridos nas conexões (linha de derivação e linhas laterais) melhorando e eficiência do uso da água na irrigação.

f) Espaçamento entre emissores - Observase, na tabela 2, que os espaçamentos entre os emissores encontram-se dentro do padrão aceitável pela norma para os quatro modelos. Cabe observar que o modelo Twin Plus não apresentou variação de espaçamentos entre os emissores gotejadores.

g) Resistência à pressão à temperatura ambiente e à temperatura de $40^{\circ} \mathrm{C}$ - Nos ensaios de resistência à pressão hidráulica em temperatura ambiente e à pressão de 1,2 e 1,8 vez à pressão máxima, os quatro modelos de tubos gotejadores apresentaram comportamento satisfatório. Nenhum tubo gotejador rompeu-se durante os testes. $\mathrm{O}$ uso de pressão elevada durante os 60 minutos do teste não prejudicou o funcionamento da membrana do gotejador autocompensante, mesmo quando foi utilizada água aquecida (Tabela 3 ).

h) Resistência à tração - Na resistência à tração com tensão de $160 \mathrm{~N}$ e $180 \mathrm{~N}$, todos os modelos ficaram dentro do padrão exigido pela norma, como pode ser observado na tabela 4 , ou seja, a vazão após o período de condicionamento dos tubos gotejadores sujeitos as duas tensões não danificou o emissor, mantendo a variação da vazão $\left(\Delta \mathrm{q}_{\mathrm{m}} \%\right)$, na pressão nominal, dentro dos limites aceitáveis
$( \pm 10 \%)$. Nos quatro modelos ensaiados, nenhum apresentou rompimento. Pode-se afirmar, de uma maneira geral, que o material usado como matériaprima na fabricação dos tubos gotejadores, bem como o processo de fabricação, é de boa qualidade, atendendo satisfatoriamente os irrigantes brasileiros. $\mathrm{O}$ ensaio de resistência à tração constitui-se em uma ferramenta rápida para diagnosticar se o tubo emissor pode ser removido e reinstalado.

De uma maneira geral, os produtos ensaiados e ofertados no mercado brasileiro apresentaram bom desempenho de acordo com a norma.

\section{CONCLUSÃO}

Os coeficientes de variação de fabricação para todos os tubos emissores avaliados foram abaixo de 0,07, permitido pela norma ABNT NBR ISO 9261:2006. Os valores dos expoentes da equação potencial (vazão x pressão) foram próximos a zero, como preconiza a função operacional dos gotejadores autocompensantes.

Tabela 3 - Resistência dos tubos gotejadores à pressão hidráulica quando operando a temperatura ambiente $\left(R_{t 1,2}, R_{t 1,8}\right)$ e aquecida $\left(40{ }^{\circ} \mathrm{C}\right)\left(\mathrm{R}_{\mathrm{t} 40}\right)$, sendo $\mathrm{R}_{\mathrm{t} 1,2}$ tubos operando $120 \%$ da pressão máxima, $\mathrm{R}_{\mathrm{t} 1,8}$ tubos operando a $180 \%$ da pressão máxima e $\mathrm{R}_{\mathrm{t} 40}$ tubos operando com água a $40^{\circ} \mathrm{C}$ e a pressão nominal.

\begin{tabular}{|c|c|c|c|c|c|c|}
\hline \multirow{2}{*}{ Modelo } & \multicolumn{2}{|c|}{------ $\mathrm{R}_{\mathrm{t} 1,2}$------- } & \multicolumn{2}{|c|}{------ $\mathrm{R}_{\mathrm{t1}, 8}$------ } & \multicolumn{2}{|c|}{------ Rt 40 ----- } \\
\hline & $\mathrm{L} \mathrm{h}^{-1}$ & $\Delta \mathrm{q}_{\mathrm{m}} \%{ }^{1}$ & $\mathrm{~L} \mathrm{~h}^{-1}$ & $\Delta \mathrm{q}_{\mathrm{m}} \%^{1}$ & $\mathrm{~L} \mathrm{~h}^{-1}$ & $\Delta \mathrm{q}_{\mathrm{m}} \%^{1}$ \\
\hline Amanco & 0,007 & 0,292 & 0,006 & 0,278 & $-0,100$ & $-4,231$ \\
\hline Naan PC & 0,003 & 0,086 & 0,000 & 0,005 & $-0,077$ & $-2,117$ \\
\hline Ram & $-0,006$ & $-0,251$ & 0,004 & 0,173 & $-0,031$ & $-1,318$ \\
\hline Twin Plus & $-0,015$ & $-0,770$ & $-0,004$ & $-0,171$ & $-0,095$ & $-4,510$ \\
\hline
\end{tabular}

Ciência Rural, v.45, n.8, ago, 2015. 
Tabela 4 - Resistência dos tubos gotejadores à tração de $160 \mathrm{~N}\left(\mathrm{R}_{\mathrm{t} 160}\right)$ e $180 \mathrm{~N}\left(\mathrm{R}_{\mathrm{t} 180 \mathrm{~N}}\right)$.

\begin{tabular}{|c|c|c|c|c|c|c|c|c|}
\hline \multirow{2}{*}{ Modelo } & \multirow[b]{2}{*}{$\mathrm{mm}$} & \multirow[b]{2}{*}{$\Delta \mathrm{L} \%^{1}$} & \multirow[b]{2}{*}{$\mathrm{L} \mathrm{h}^{-1}$} & \multirow[b]{2}{*}{$\Delta \mathrm{q}_{\mathrm{m}} \%^{1}$} & \multirow[b]{2}{*}{$\mathrm{mm}$} & \multirow[b]{2}{*}{$\Delta \mathrm{L} \%$} & \multirow[b]{2}{*}{$\mathrm{L} \mathrm{h}^{-1}$} & \multirow[b]{2}{*}{$\Delta \mathrm{q}_{\mathrm{m}} \%$} \\
\hline & & & & & & & & \\
\hline Amanco & 151,0 & 0,67 & 0,004 & 0,187 & 151,0 & 0,67 & 0,006 & 0,214 \\
\hline Naan PC & 150,5 & 0,33 & 0,002 & 0,053 & 151,5 & 1 & 0,005 & 0,142 \\
\hline Ram & 151,0 & 0,67 & 0,007 & 0,312 & 152,0 & 1,33 & 0,007 & 0,282 \\
\hline Twin Plus & 150,5 & 0,33 & $-0,001$ & $-0,068$ & 151,0 & 0,67 & 0,004 & 0,205 \\
\hline
\end{tabular}

${ }^{1} \Delta \mathrm{L} \%=$ variação do comprimento do tubo gotejador após condicionamento (aceitável $\pm 5 \%$ ); $\Delta \mathrm{q}_{\mathrm{m}} \%$ (aceitável $\pm 10 \%$ ) (ABNT NBR ISO 9261:2006).

\section{REFERÊNCIAS}

ABNT (ASSOCIAÇÃO BRASILEIRA DE NORMAS TÉCNICAS). NBR ISO 9261: equipamentos de irrigação agrícola. Emissores e tubos emissores. Especificação e métodos de ensaio. São Paulo, 2006. 17p.

ANDRADE, C. de L.T de.; BORGES JUNIOR, J.C.F. Seleção do método de irrigação. In: ALBUQUERQUE, P.E.P. de; DURÃES, F.O.M. (Eds.). Uso e manejo de irrigação. Brasília: EMBRAPA, 2008. Cap.7, p.317-399.

FRIZZONE, J.A.; DOURADO NETO, D. Avaliação de sistemas de irrigação e análise econômica. In: MIRANDA, J.H. de; PIRES, R.C. de M. (Eds.). Irrigação. Jaboticabal: FUNEP, 2003. V.2, cap. 15, p.573-652.

FRIZZONE, J.A. et al. Microirrigação: gotejamento e microaspersão. Maringá: Eduem, 2012. 356p.

GOMES, H.P. Engenharia de irrigação: hidráulica dos sistemas pressurizados, aspersão e gotejamento. 2 ed. rev. ampl. Campina Grande: UFPB, 1997. 390p.
KELLER, J.; BLIESNER, R.D. Sprinkle and trickle irrigation. New York: van Nostrand Reinhold, 1990. 652p.

MANTOVANI, E.C. et al. Irrigação: princípios e métodos. 3.ed. Viçosa: UFV, 2012. 355p.

PIZARRO CABELLO, F. Riegos localizados de alta frecuencia (RLAF): goteo, microaspersión, exudación. 3.ed. rev. ampl. Madrid: Mundi Prensa, 1996. 513p.

PRADO, G. do et al. Avaliação técnica de dois tipos de emissores empregados na irrigação localizada. Revista Brasileira de Agricultura Irrigada, Fortaleza, v.8, n.1, p.12-25, jan. 2014. Disponível em: <http:// www.inovagri.org.br/revista/index.php/rbai/article/view/193>. Acesso em: 25 abr. 2014. doi: 10.7127/rbai.v8n100204.

RODRIGO LOPEZ, R.J. et al. Riego localizado. 2.ed. Madrid: Mundi Prensa, 1997. 405p.

SOUZA, W.J. et al. Irrigação localizada subsuperficial: gotejador convencional e novo protótipo. Revista Brasileira de Engenharia Agrícola e Ambiental, Campina Grande, v.16, n.8, p.811-819, ago. 2012. Disponível em: <http://www.scielo.br/pdf/rbeaa/v16n8/ a01v16n8.pdf> . Acesso em: 10 abr. 2014. doi: 10.1590/S141543662012000800001 . 